\title{
EVALUACIÓN DEL NIVEL DE ADOPCIÓN DE INTERNET EN LAS UNIVERSIDADES CHILENAS EN BASE AL MODELO EMICA
}

\section{EVALUATION OF THE INTERNET ADOPTION LEVEL IN CHILEAN UNIVERSITIES BASED ON THE EMICA MODEL}

\author{
Narciso Cerpa ${ }^{1} \quad$ Andrés Ruiz-Tagle $^{2} \quad$ Carolina Cabrera $^{3}$ \\ Pamela Hadweh $^{4} \quad$ Fabián Vergara $^{5}$ \\ Recibido 1 de diciembre de 2006, aceptado 24 de julio de 2007 \\ Received: December 1, 2006 Accepted: July 24, 2007
}

\begin{abstract}
RESUMEN
Este estudio investiga el grado de adopción de las tecnologías de Internet en las universidades chilenas, como sistema de promoción y coordinación de sus actividades, considerando el rol de esta tecnología en la comunicación efectiva con los diferentes actores sociales que se relacionan con la universidad, tales como empresas, instituciones, personas y postulantes. Esta plataforma tecnológica también juega un rol a diario y muy importante en la comunicación entre miembros internos: administrativos, académicos y alumnos. El estudio consistió en el análisis de los sitios Web de todas las universidades chilenas bajo la metodología, eMICA, un modelo extendido de adopción de comercio electrónico en Internet [3]. Los resultados de este estudio indican que las universidades en general hacen un buen uso de las tecnologías de Internet que permite a una gran mayoría $(98,3 \%)$ de éstas realizar promoción y suministro de información con un alto grado de sofisticación e interactividad (etapa 2, nivel 3 del modelo eMICA). Además, gran parte (75\%) de las universidades nacionales muestran un alto grado de adopción de tecnologías de Internet, ya que cuentan con aplicaciones (administrativas, académicas, promocionales, etc.) que permiten hacer transacciones por medio de la Web y, por lo tanto, se encuentran en la etapa más alta, procesamiento, de acuerdo al modelo utilizado.
\end{abstract}

Palabras clave: eMICA, educación superior, sitios Web universidades, desarrollo Web, adopción de comercio electrónico, tecnología Internet, modelos de adopción.

\section{ABSTRACT}

The main objective of this study is to investigate the degree of Internet technologies adoption in by chilean universities. These universities use the Web as a promotion system and for the coordination of their operational activities, highlighting the role of this technology in the effective communication between the different social actors that interact with the university, namely, enterprises, institutions, people and potential students. This Web-based platform plays also an important role in daily communication among administrative staff, faculty, and students. This study analyses all the chilean universities' websites, applying the Extended Model of Internet Commerce Adoption (eMICA) [3]. The results from this study show that in general, the universities make a wide use of Internet technologies, which allows most of these institutions (98.3\%), to do their promotion and also provide information with a high degree of sophistication and interactivity (stage 2, level 3 of the eMICA model). A large percentage (75\%) of the Chilean universities show a high degree of Internet technologies adoption, since they have application systems (administrative, academic, promotional, etc.), that support the execution of transactions via the Web, therefore, they are in the highest stage of the eMICA model, transaction processing.

Keywords: eMICA, higher education, universities' websites, Web development, e-Commerce adoption, Internet technology, adoption models.

\footnotetext{
Facultad de Ingeniería. Universidad de Talca. Merced 437, Curicó, Chile. E-mail: ncerpa@utalca.cl

Facultad de Ciencias Empresariales. Universidad de Talca. 2 Norte 685. Talca, Chile. E-mail: aruiz-tagle@utalca.cl

3 Facultad de Ciencias Empresariales. Universidad de Talca. 2 Norte 685, Talca, Chile. E-mail: cmcabrera@banmedica.cl

Facultad de Ciencias Empresariales. Universidad de Talca. 2 Norte 685, Talca, Chile. E-mail: phadweh@santandersantiago.cl

Facultad de Ingeniería. Universidad de Talca. Merced 437, Curicó, Chile. E-mail: favergara@utalca.cl
} 


\section{INTRODUCCIÓN}

Los cambios tecnológicos han transformado la naturaleza de las economías y de la comunidad global [12]. Un ejemplo de ello es Internet, que es considerada un medio diferencial en términos de rapidez, funcionalidad y contenidos de información [13]. Una prueba es el cambio radical en la relación cliente-empresa que se ha transformado en una interacción en línea a través de Internet [19, 21]. Los fundamentos de estos cambios están afectando a la industria y a la comunidad, no quedando el sector educativo ajeno a ellos.

Internet se ha convertido en un medio importante para complementar y desarrollar el aprendizaje, pero no sólo en este ámbito la presencia de Internet ha provocado cambios, sino que, además, se ha convertido en una importante herramienta de marketing para las instituciones educacionales, un medio de comunicación masivo más económico que los existentes que elimina las barreras geográficas y es preferido por los postulantes que buscan información sobre las universidades.

Durante los últimos 20 años la educación superior se ha vuelto más competitiva, privatizada, segmentada y conducida por el mercado. Factores que la han hecho sensible al impactante desarrollo de Internet causado por la aparición de su instrumento más popular, la World Wide Web (WWW) que no ha dejado prácticamente ningún área que no se haya visto afectada por algún tipo de cambio, siendo algunos de ellos muy profundos [9].

Si bien en la educación superior las transformaciones influenciadas directamente por Internet son más recientes que en otros sectores $[1,16]$, es un hecho que en este momento la educación universitaria se encuentra inmersa en una corriente de cambios fomentados tecnológicamente, cuyo alcance e importancia fueron previstos hace algunos años $[14,17]$.

De acuerdo a lo expuesto en el párrafo anterior, en la actualidad la presencia de la WWW en el proceso de aprendizaje se puede clasificar como una estrategia complementaria desde el punto de vista del comercio electrónico basado en Internet [12], ya que puede ser de diversa magnitud: desde la mera posibilidad de descarga de contenidos docentes en línea hasta la comunicación asincrónica (foro o lista de correo) con otros estudiantes o con el profesor, pasando por la visualización en línea de clases magistrales o la autocorrección de ejercicios y exámenes.
La principal clasificación de la enseñanza basada en la WWW [18] se refiere a si ésta es un medio que complementa la enseñanza presencial tradicional o si, por el contrario, el alumno centra su formación exclusivamente en el entorno WWW y no tiene contacto directo con otros estudiantes o con el profesor (entorno educativo virtual). En el caso de las universidades nacionales, que son el objeto de estudio, no solamente éste ha sido el fin que persiguen al implementar páginas Web, sino que además se considera como un medio de marketing, comunicación y difusión para dichas instituciones.

En base a lo expuesto anteriormente, es importante conocer el grado de adopción de Internet de las universidades chilenas y principalmente determinar el nivel de desarrollo alcanzado por los servicios que estas instituciones prestan a sus clientes potenciales y actuales. Para lograr este objetivo, se utilizó el modelo extendido de adopción de comercio electrónico en Internet (eMICA) [3], el cual ha sido utilizado anteriormente para analizar la adopción de comercio electrónico en la industria del turismo en Nueva Zelanda, Australia y Chile [2-5, 7].

La importancia de conocer la evolución de las páginas Web de las universidades chilenas radica en las siguientes consideraciones:

- La primera de ellas es la gran penetración de Internet en Chile, liderando Latinoamérica, con un 35\% de usuarios de Internet, dicho porcentaje es elevado si se compara con países como Argentina, que posee un $15 \%$. Es importante mencionar que Chile se encuentra en un nivel similar al de Italia y España [10].

- Una segunda consideración apunta a que la educación es la tercera actividad más desarrollada por los chilenos en Internet [11].

- La tercera razón es que nunca se ha realizado un estudio para medir la adopción de Internet de las universidades chilenas, por lo tanto, corresponde a un campo inexplorado que puede dar pie a muchos otros estudios relacionados con el tema.

- Por último, este estudio permite comparar el nivel de evolución de los sitios Web de las universidades chilenas y así conocer su posicionamiento frente a la competencia y determinar los servicios que les falta implementar.

Para la medición de los niveles de adopción de Internet en las universidades chilenas, se analizaron los sitios Web de las 60 instituciones de educación superior existentes en 
el país, de las cuales 25 pertenecen al consejo de rectores y 35 tienen el carácter de privadas. La estratificación de universidades antes mencionada surge en Chile en diciembre de 1980 cuando se dicta el Decreto Ley N 3.541 que hace desaparecer las Sedes fuera de Santiago pertenecientes a las dos universidades estatales existentes a esa fecha (Universidad de Chile y Universidad Técnica del Estado, actualmente Universidad de Santiago de Chile). Sobre la base de éstas se crean instituciones que, junto a las sedes que la Pontificia Universidad Católica de Chile posee fuera de Santiago, se conocen como universidades regionales y/o derivadas. Asimismo, esta nueva legislación permitió la creación de universidades privadas, que deben constituirse como personas jurídicas de derecho privado sin fines de lucro y que no están adscritas al Consejo de Rectores, dando origen a una gran expansión del Sistema Universitario Nacional [6].

En términos metodológicos, inicialmente se realiza una revisión de modelos que permitiera determinar el grado de adopción de Internet. De los modelos consultados, se determina que la mayor aplicabilidad la entregaba el modelo eMICA. En base a él, posteriormente se estudian los servicios que entregan las 60 universidades chilenas en sus respectivos sitios Web y, por último, se efectúa una comparación entre sus sitios.

\section{MARCO TEÓRICO}

De acuerdo a lo expuesto en la sección anterior, es importante evaluar el grado de adopción de Internet de las universidades chilenas y principalmente determinar el nivel de desarrollo alcanzado por los servicios que estas instituciones prestan a sus clientes potenciales y actuales. Para ello se analizaron varios modelos aplicados a diversas industrias, que plantean objetivos similares al modelo eMICA finalmente utilizado.

Uno de los modelos analizados se centra en la estrategia de diseñar sitios Web para organizaciones [15]. Su desarrollo se consiguió analizando detalladamente las formas de difusión de la tecnología Web en dos organizaciones americanas de computadoras y determinó cómo dichas entidades establecieron sus sitios, lo cual permitió el reconocimiento de puntos relevantes, los que posteriormente se transformaron en niveles. Estos niveles son: departamento de tecnologías de información, gerencia superior, comité pertinente (de carácter informal), crisis de funcionamiento y aceleradores de adopción. Este modelo no se utiliza en este estudio debido a que en este caso se analizan las 60 universidades existentes a lo largo de Chile, las cuales pueden tener diferencias estructurales y culturales, a pesar de que se encuentran en el mismo territorio, situaciones que este modelo no logró resolver a plenitud en los estudios llevados a cabo [22].

En [20] definen un nuevo modelo que se centra en las etapas por las que pasa la creación e implantación de una página Web: Información, transacción y operacional. Debido a que este modelo permite sólo clasificar las páginas dentro de estos tres segmentos, sus definiciones son demasiado amplias para conocer el nivel en que se encuentra la adopción de Internet en las universidades chilenas.

En otro estudio [7] propone una metodología para la evaluación y análisis de sitios Web de gobierno, la que contiene seis áreas: audiencias, declaración del propósito, objetivos, especificación de la página, información del dominio, y presentación de la Web. Sin embargo, esta metodología es limitada en su uso, debido a sus amplios términos y a la carencia de un soporte claro de referencias del modelo o método, haciendo las evaluaciones de una gran cantidad de sitios Web una tarea impracticable.

\section{Modelo MICA}

El modelo de adopción del comercio de Internet, MICA [2], fue desarrollado originalmente para un estudio en la industria australiana de la fabricación de metal después de tomar en consideración ciertos modelos de evaluación de sitios Web de otros autores. El modelo propone que al crear sitios comerciales, las organizaciones comienzan estableciendo sólo una presencia en la Web y posteriormente desarrollan funcionalidades a medida que aumenta su experiencia en el uso de Internet.

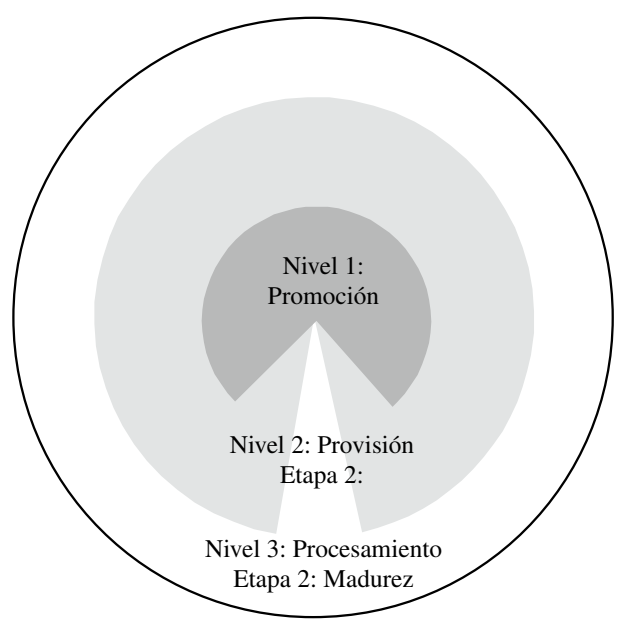

Figura 1. Modelo MICA. 
El proceso de promoción es el nivel inicial de desarrollo; consiste en información estática que sirve principalmente para promover y publicar las ofertas del negocio. Dentro de este nivel, una primera etapa se presenta normalmente con la información básica de la compañía: dirección, información de contacto e información de carácter general sobre los productos y los servicios ofrecidos. Se considera que la organización está en el segundo nivel del modelo (provisión), si el sitio va más allá de simplemente tener una presencia en la Web. El tercer nivel (procesamiento) presenta procesos transaccionales.

MICA fue modificado debido a que era muy amplio, especialmente en el nivel 2 (provisión), por lo que se creó el modelo extendido de adopción del comercio en Internet, eMICA (2000), que fue utilizado en las industrias de turismo de Nueva Zelanda [8], Australia $[5,8]$ y Chile [4].

\section{Modelo eMICA}

El modelo extendido de adopción de comercio en Internet, eMICA (2000), agrega varias capas de sofisticación, funcionalidad e innovación dentro de las tres etapas principales, para acomodarse a la amplia gama del desarrollo del comercio en Internet, como se muestra en la figura 2.

En la tabla 1 se detalla cada una de las etapas con sus respectivos niveles adaptados para realizar el estudio de los sitios Web de las universidades chilenas.

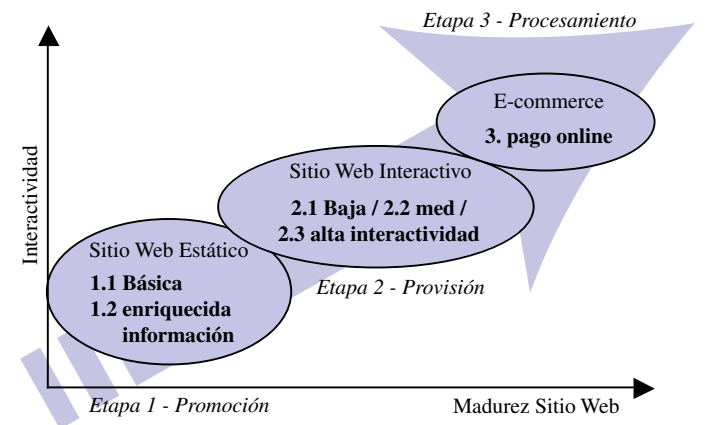

Figura 2. Modelo eMICA [3, 8].

El proceso de promoción es la etapa inicial de desarrollo, consiste en información estática que sirve principalmente para promover y publicar las ofertas del negocio. Dentro de esta etapa, un primer nivel se presenta normalmente con la información básica sobre la compañía, su dirección, información de contacto e información de carácter general sobre productos y servicios ofrecidos. Un segundo nivel presenta un grado más enriquecido de información, entre los que se consideran: e-mail de contacto e información sobre actividades de la organización.

Tabla 1. Detalle de atributos considerados.

\begin{tabular}{|c|c|c|}
\hline Etapa & Nivel & Atributos \\
\hline \multirow{7}{*}{ Promocion } & \multirow{5}{*}{ Nivel 1} & Nombre universidad \\
\hline & & Casilla \\
\hline & & Dirección física \\
\hline & & Detalles contacto \\
\hline & & Área negocios \\
\hline & \multirow[b]{2}{*}{ Nivel 2} & E-mail de contacto \\
\hline & & $\begin{array}{l}\text { Información sobre actividades } \\
\text { de la Compañía. }\end{array}$ \\
\hline \multirow{15}{*}{ Provisión } & \multirow{2}{*}{ Nivel 1} & Catálogo básico de servicios \\
\hline & & Formulario de consulta \\
\hline & \multirow{7}{*}{ Nivel 2} & Catálogo de alto nivel \\
\hline & & Ayuda al cliente \\
\hline & & Buscadores \\
\hline & & Características de la región \\
\hline & & Hiperlinks \\
\hline & & Valor agregado \\
\hline & & Mapas y rutas \\
\hline & \multirow{6}{*}{ Nivel 3} & Chat \\
\hline & & Foro \\
\hline & & Multimedia \\
\hline & & Noticias vía e-mail \\
\hline & & Opciones de lenguaje \\
\hline & & E-mail para clientes \\
\hline \multirow{3}{*}{ Procesamiento } & & Transacciones \\
\hline & & Estado de orden \\
\hline & & Interacción con el servidor \\
\hline
\end{tabular}

La segunda etapa descrita por el modelo eMICA, hace referencia a un sitio Web que evoluciona a un sistema de información dinámico. Dentro de esta etapa se identifican tres niveles: uno de baja interactividad caracterizado por catálogos en línea, links a información detallada; uno de media interactividad con catálogos más completos del producto o servicio, ayuda en línea para los usuarios, así como valor agregado y un nivel de alta interactividad del sitio que incluye chat, foros de discusión, multimedia y boletines por e-mail a pedido. 
La tercera etapa del modelo eMICA se alcanza cuando el sitio Web tiene una madurez funcional que le permite realizar transacciones en línea. Esto requiere la identificación del usuario y un grado más alto de seguridad que en las etapas anteriores. En esta etapa los usuarios podrán comprar productos y servicios a través de la Web y mantener un perfil personal.

A continuación se describen los servicios pertenecientes a los atributos especificados en el cuadro anterior (tabla 1).

\section{Etapa 1: Promoción}

Nivel 1: Información básica.

- Nombre: corresponde a nombre de la universidad.

- Casilla: dirección de correo postal.

- Dirección física: dirección física de la universidad $\mathrm{y}$ de sus sedes en el caso que tenga.

- Detalles contacto: teléfono y fax.

- Área negocios: descripción del tipo de actividades que se desarrollan en la educación superior: docencia, investigación y extensión.

Nivel 2: Información Avanzada

- E-mail de contacto: dirección de correo electrónico para poder comunicarse con la universidad o algún departamento en particular.

- Información sobre actividades de la compañía: Información sobre programas de pregrado, postgrado y centros tecnológicos.

\section{Etapa 2: Provisión}

Nivel 1: Nivel bajo de interactividad

- Catálogo básico de servicios: presentación de las carreras de la universidad, perfil profesional, malla curricular, puntajes requeridos para el ingreso en una página estática.

- Formulario de consulta: un formulario en línea para realizar cualquier tipo de consulta o duda.

Nivel 2: Nivel medio de interactividad

- Catálogo de alto nivel: páginas por carrera, catálogo propio para admisión.

- Ayuda al cliente: en la forma de preguntas frecuentes y mapa del sitio.

- Buscadores: dentro de la página de la universidad (personas, documentos, links, etc.).
- Características de la región: información sobre particularidades de la región en la que se encuentra ubicada la universidad, clima, vegetación, lugares turísticos, etc.

- Hiperlinks: información adicional o los servicios que agregan valor a la experiencia del cliente como: universia, Consejo de Rectores, google, entre otros.

- Valor agregado: cosas que no están directamente relacionadas con la educación como una bolsa de trabajo, disco virtual, cifras económicas, etc.

- Mapas y rutas: mapas que permiten llegar al lugar donde se encuentra el campus y mapas de ubicación dentro de la universidad.

Nivel 3: Nivel alto de interactividad

- Chat: sistema para conversar de manera escrita y en tiempo real con otros usuarios de Internet, quienes pueden leer el mensaje casi inmediatamente de ser enviado y responder con la misma brevedad.

- Foro: herramienta de Internet que permite a los usuarios expresar sus opiniones, respondiendo a una pregunta o escribiendo sus comentarios, los cuales podrán ser leídos por cualquier otro usuario de la red.

- Multimedia: material digitalizado que combina textos, gráficos, imagen fija y en movimiento, así como sonido.

- Noticias vía mail: noticias de carácter institucional que es enviada a la cuenta de correo electrónico de los alumnos o funcionarios de las universidades.

- Opciones de lenguaje: permite que la página se vea y se pueda navegar en ella en otro idioma.

- E-mail para clientes: la posibilidad que otorga la universidad de que los alumnos puedan crear una cuenta de correo electrónico de la universidad.

\section{Etapa 3: Procesamiento}

- Transacciones: en este ítem se consideraron tanto las financieras (pago en línea de la matrícula, de las mensualidades, de los cursos o postgrados) como las no financieras (solicitud de certificados, evaluación docente, inscripción de ramos, pruebas en línea).

- Estado de orden: si la orden cambia de estado, pedido, en trámite, entregado/evaluado (si sólo se recibe, no tiene estado).

- Interacción con el servidor: corresponde a transacciones de consulta, esto es, acceso a base de datos (ficha personal, notas parciales e históricas, etc.). 


\section{METODOLOGÍA}

Para llevar a cabo el presente estudio, en una primera instancia se buscó información del modelo eMICA, el que fue aplicado en diversos países para la industria del turismo [3-5, 8]. Posteriormente, para determinar si dicho modelo era el más adecuado de aplicar, se consultaron los diversos modelos expuestos en el Marco Teórico, para luego evaluar la factibilidad de su aplicación.

Por sus características de adaptabilidad a diversas industrias, se determinó que la versión extendida del modelo MICA (eMICA) es la que mejor se ajusta al propósito del estudio, ya que permite evaluar los sitios Web desde un nivel promocional, pasando por el suministro de información y servicio, llegando hasta procesos transaccionales, indicando así en qué nivel de adopción de este instrumento se encuentran las universidades chilenas. Además, la aplicación del modelo a esta investigación permitió evaluar su pertinencia para el sector universitario.

Para lo anterior se analizaron los sitios Web (Internet e Intranets) de 60 universidades chilenas durante el primer semestre del año 2005. Para el acceso a las Intranets se solicitaron claves de acceso a las áreas de informática y en ocasiones pidió la colaboración de académicos y estudiantes de dichas instituciones. Este proceso permitió generar una imagen global del uso de la Web en las universidades chilenas.

Para evitar dobles lecturas durante la evaluación de los sitios, se procedió a la creación de estándares que definieran claramente las características y funcionalidades a analizar. Este proceso permitió determinar claramente la presencia o ausencia de un determinado elemento al analizar en detalle las características y funciones de cada sitio Web, para posteriormente agruparlos de acuerdo a niveles de funcionalidad, interactividad y sofisticación. De esta forma se facilitó la categorización de cada sitio según las etapas y niveles propuestos por el modelo eMICA y por ende la determinación del grado de adopción de cada sitio Web universitario.

No obstante lo anterior, es necesario señalar que debido a que el modelo eMICA responde a un proceso de adopción gradual de Internet, es posible encontrar casos en los cuales el ingreso a la Web es explosivo y no paso a paso, incorporando elementos propios de las etapas y niveles más altos del modelo. En estos casos, el posicionamiento otorgado es del más alto nivel, aun cuando pueda existir ausencia de algún ítem perteneciente a los niveles o etapas básicas del modelo. En otras palabras, las universidades que se encuentren en la etapa de procesamiento no necesariamente deben contar con la presencia de las veinticinco variables evaluadas.

\section{RESULTADOS DE LA EVALUACIÓN DE LOS SITIOS WEB}

Los datos obtenidos de la revisión de los 60 sitios Web de las universidades chilenas permitieron elaborar la tabla 2 , donde se muestran las etapas del modelo eMICA con sus respectivos niveles y la frecuencia de cada atributo en el total de sitios analizados.

Tabla 2. Frecuencia de atributos.

\begin{tabular}{|c|c|c|c|c|}
\hline Etapa & Nivel & Variables & $\begin{array}{l}\text { Cant. } \\
\text { Univ. }\end{array}$ & $\%$ \\
\hline \multirow[t]{7}{*}{ Promoción } & \multirow[t]{5}{*}{1} & Nombre universidad & 60 & 100,0 \\
\hline & & Casilla & 19 & 31,7 \\
\hline & & Dirección física & 59 & 98,3 \\
\hline & & Detalles contacto & 60 & 100,0 \\
\hline & & Área negocios & 60 & 100,0 \\
\hline & \multirow[t]{2}{*}{2} & E-mail de contacto & 59 & 98,3 \\
\hline & & \begin{tabular}{|l|} 
Inf. sobre actividades \\
de las universidades
\end{tabular} & 59 & 98,3 \\
\hline \multirow[t]{14}{*}{ Provisión } & \multirow[t]{2}{*}{1} & $\begin{array}{l}\text { Catálogo básico de } \\
\text { servicios }\end{array}$ & 60 & 100,0 \\
\hline & & Formulario de consulta & 30 & 50,0 \\
\hline & \multirow[t]{6}{*}{2} & Catálogo de alto nivel & 35 & 58,3 \\
\hline & & Ayuda al cliente & 31 & 51,7 \\
\hline & & Buscadores & 23 & 38,3 \\
\hline & & Hiperlinks & 49 & 81,7 \\
\hline & & \begin{tabular}{|l} 
Valor agregado \\
\end{tabular} & 32 & 53,3 \\
\hline & & Mapas y rutas & 20 & 33,3 \\
\hline & \multirow[t]{6}{*}{3} & Chat & 12 & 20,0 \\
\hline & & Foro & 26 & 43,3 \\
\hline & & Multimedia & 55 & 91,7 \\
\hline & & \begin{tabular}{|l|} 
Noticias vía mail \\
\end{tabular} & 25 & 41,7 \\
\hline & & \begin{tabular}{|l|} 
Opciones de lenguaje \\
\end{tabular} & 6 & 10,0 \\
\hline & & E-mail para clientes & 51 & 85,0 \\
\hline \multirow{3}{*}{ Procesamiento } & & Transacciones & 42 & 70,0 \\
\hline & & Estado de orden & 37 & 61,7 \\
\hline & & $\begin{array}{l}\text { Interacción con el } \\
\text { servidor }\end{array}$ & 42 & 70,0 \\
\hline
\end{tabular}

Para determinar en cuál de las etapas del modelo las universidades chilenas tienen un mejor desempeño, a continuación se consideran los porcentajes alcanzados 
para cada atributo evaluado, obteniéndose así el desempeño promedio de las universidades en cada etapa del modelo.

Tabla 3. Desempeño promedio de las universidades en cada etapa.

\begin{tabular}{|l|c|}
\hline Etapa & \% Presencia Promedio \\
\hline Promoción & 89,5 \\
\hline Provisión & 54,2 \\
\hline Procesamiento & 67,2 \\
\hline
\end{tabular}

Si calculamos el promedio de las variables observadas por etapa, como se muestra en la tabla 3, la etapa 2 "Provisión" es aquella en que las universidades chilenas muestran un menor grado de penetración, alcanzando tan sólo el 54,2\%. Sin embargo, esto está influenciado fuertemente por dos variables (chat y opciones de lenguaje) que hacen decaer fuertemente el promedio. Si estas no se consideran, el promedio se eleva inmediatamente a un $63,2 \%$ de cumplimiento, muy similar al grado de cumplimiento de la etapa $3(67,2 \%)$.

Si bien es cierto la variable chat puede no ser muy relevante en cuanto a la importancia de estar o no presente en el sitio, la variable opciones de lenguaje sí lo es ya que se trata de una componente estratégica importante de cada institución si es que está inserta en una red internacional de universidades y que a la vez se transforma en un elemento distintivo del sitio Web, dado el escaso nivel de presencia de esta variable.

No obstante lo anterior, es necesario señalar que la ausencia de una variable en una etapa determinada no implica que una institución no pueda considerarse como parte de una etapa superior de adopción de Internet, es decir, la escasa presencia de opciones de lenguaje (etapa 2) en los sitios no impedirá que la universidad que no la posea pueda ser considerada en la etapa 3.

\section{Análisis del nivel de adopción total}

Es destacable que todos los sitios analizados sobrepasan la primera etapa del modelo, en la que se incluyen cualidades y funcionalidades básicas de un sitio Web.

En la tabla 4 se observa que de las 60 universidades chilenas, un $25 \%$ está en la etapa 2 de provisión. De ellas un 1,7\% están en el nivel 2 (nivel medio de interactividad), un $23,3 \%$ en el tercer nivel (alto nivel de interactividad) y prácticamente la mayoría (75\%) se encuentra concentrado en la etapa 3 de procesamiento (transaccional).
Tabla 4. Nivel de adopción total.

\begin{tabular}{|l|c|c|c|}
\hline \multicolumn{1}{|c|}{ Etapa } & Nivel & Cant. de Universidades & \% \\
\hline \multirow{3}{*}{ Promoción } & $\mathbf{1}$ & 0 & 0 \\
\cline { 2 - 4 } & $\mathbf{2}$ & 0 & 0 \\
\hline \multirow{3}{*}{ Provisión } & $\mathbf{1}$ & 0 & 0 \\
\cline { 2 - 4 } & $\mathbf{2}$ & 1 & 1,7 \\
\cline { 2 - 4 } & $\mathbf{3}$ & 14 & 23,3 \\
\hline Procesamiento & & 45 & 75 \\
\hline TOTAL & & 60 & 100 \\
\hline
\end{tabular}

Por lo tanto, la industria de la educación superior en Chile, específicamente de las universidades, son un sector muy avanzado en la adopción de Internet, que ha alcanzado altos niveles de funcionalidad e interactividad dentro de sus sitios Web, facilitando a los actuales y potenciales clientes una amplia gama de servicios relacionados con la educación y en algunos casos satisfaciendo necesidades que no están directamente relacionadas con ésta.

Para tener una visión más detalla, a continuación se clasifican los resultados obtenidos de acuerdo a diversos criterios.

Análisis del nivel de adopción de las universidades privadas no tradicionales

De las 60 universidades chilenas, 35 se clasifican como privadas.

Tabla 5. Análisis del nivel de adopción de las universidades privadas.

\begin{tabular}{|l|c|c|c|}
\hline \multicolumn{1}{|c|}{ Etapa } & Nivel & Cant. de Universidades & \% \\
\hline \multirow{2}{*}{ Promoción } & $\mathbf{1}$ & 0 & 0 \\
\cline { 2 - 4 } & $\mathbf{2}$ & 0 & 0 \\
\hline \multirow{3}{*}{ Provisión } & $\mathbf{1}$ & 0 & 0 \\
\cline { 2 - 4 } & $\mathbf{2}$ & 0 & 0 \\
\cline { 2 - 4 } & $\mathbf{3}$ & 13 & 37,1 \\
\hline Procesamiento & & 22 & 62,9 \\
\hline TOTAL & & 35 & 100 \\
\hline
\end{tabular}

En la tabla 5 se observa que dentro de esta categoría el 37,1\% alcanza el nivel 3 de alta interactividad de la etapa 2 y el $62,9 \%$ la etapa 3 de procesamiento, es decir, poseen una gran funcionalidad, llegando a altos niveles transaccionales, que van desde el pago de matrículas y aranceles vía online hasta la solicitud de certificados, permitiendo en algunos casos que los usuarios puedan consultar el estado de sus transacciones y acceder a esta información en cualquier momento. 
Análisis del nivel de adopción de las universidades pertenecientes al Consejo de Rectores

Del total de universidades chilenas, 25 pertenecen al Consejo de Rectores. En la tabla 6 se observa que dentro de esta categoría el $4 \%$ de las universidades se encuentra en la etapa 2 , nivel 2 , otro $4 \%$ se encuentra en la etapa 2 , nivel 3 y el $92 \%$ restante está en la etapa más avanzada del modelo, procesamiento.

Tabla 6. Análisis del nivel de adopción de las universidades pertenecientes al Consejo de Rectores.

\begin{tabular}{|l|c|c|c|}
\hline & Nivel & Cant. de Universidades & \% \\
\hline \multirow{2}{*}{ Promoción } & $\mathbf{1}$ & 0 & 0 \\
\cline { 2 - 4 } & $\mathbf{2}$ & 0 & 0 \\
\hline \multirow{3}{*}{ Provisión } & $\mathbf{1}$ & 0 & 0 \\
\cline { 2 - 4 } & $\mathbf{2}$ & 1 & 4 \\
\cline { 2 - 4 } & $\mathbf{3}$ & 1 & 4 \\
\hline Procesamiento & & 23 & 92 \\
\hline TOTAL & & 25 & 100 \\
\hline
\end{tabular}

Por lo tanto, las universidades del Consejo de Rectores alcanzan un alto nivel de adopción de Internet y sobrepasan en 30 puntos porcentuales a las universidades privadas que se encuentran en esta misma etapa. Es posible que la distribución geográfica de las universidades del consejo de rectores (20 en regiones y 5 en la Región Metropolitana) explique este mayor uso de Internet.

\section{Análisis del nivel de adopción de las universidades ubicadas en la Región Metropolitana}

Del total de universidades, 30 se encuentran en la Región Metropolitana, de las cuales el 66,7\% está dentro de la tercera etapa del modelo, un $30 \%$ en el nivel 3 de la etapa 2 y sólo un 3,3\% en el nivel 2 de la misma etapa (ver tabla 7).

Tabla 7. Análisis del nivel de adopción de las universidades ubicadas en la Región Metropolitana.

\begin{tabular}{|l|c|c|c|}
\hline \multicolumn{1}{|c|}{ Etapa } & Nivel & Cant. de Universidades & $\mathbf{\%}$ \\
\hline \multirow{2}{*}{ Promoción } & $\mathbf{1}$ & 0 & 0 \\
\cline { 2 - 4 } & $\mathbf{2}$ & 0 & 0 \\
\hline \multirow{3}{*}{ Provisión } & $\mathbf{1}$ & 0 & 0 \\
\cline { 2 - 4 } & $\mathbf{2}$ & 1 & 3,3 \\
\cline { 2 - 4 } & $\mathbf{3}$ & 9 & 30,0 \\
\hline Procesamiento & & 20 & 66,7 \\
\hline TOTAL & & 30 & 100 \\
\hline
\end{tabular}

Análisis del nivel de adopción de las universidades ubicadas en regiones

Del total de universidades, 30 se encuentran en regiones. De éstas, un $16,7 \%$ está dentro de la segunda etapa en su tercer nivel y el $83 \%$ en la etapa más avanzada, la de procesamiento (ver tabla 8 ).

Por lo tanto, se puede decir que las universidades ubicadas en regiones alcanzan un nivel más alto de adopción de Internet que las ubicadas en la Región Metropolitana.

Tabla 8. Análisis del nivel de adopción de las universidades ubicadas en regiones.

\begin{tabular}{|l|c|c|c|}
\hline \multicolumn{1}{|c|}{ Etapa } & Nivel & Cant. de Universidades & \% \\
\hline \multirow{3}{*}{ Promoción } & 1 & 0 & 0 \\
\cline { 2 - 4 } & 2 & 0 & 0 \\
\hline \multirow{3}{*}{ Provisión } & 1 & 0 & 0 \\
\cline { 2 - 4 } & 2 & 0 & 0 \\
\cline { 2 - 4 } & 3 & 5 & 16,7 \\
\hline Procesamiento & & 25 & 83,3 \\
\hline TOTAL & & 30 & 100 \\
\hline
\end{tabular}

Comparación del nivel de adopción de las universidades por tipo de institución (Consejo de Rectores (CR)privada) y ubicación geográfica (Región Metropolitana (RM)-regiones)

Por los resultados descritos en los puntos anteriores se hace necesaria una comparación entre los dos criterios utilizados para clasificar las universidades (ver tabla 9).

De las 25 universidades del consejo de rectores:

- 20 se encuentran en regiones, de las cuales 19 (95\%) alcanzan la etapa 3 y sólo 1 (5\%) la etapa 2 nivel 3.

- 5 se encuentran en la Región Metropolitana, de las cuales $4(80 \%)$ se encuentran en la etapa 3 y sólo 1 $(20 \%)$ en la etapa 2 nivel 2.

De las 35 universidades privadas:

- 10 se encuentran en regiones, de las cuales $6(60 \%)$ alcanzan la etapa 3 y 4 (40\%) la etapa 2 nivel 3.

- 25 se encuentran en la Región Metropolitana de las cuales $16(64 \%)$ se encuentran en la etapa 3 y 9 $(36 \%)$ en la etapa 2 nivel 3.

Por lo tanto, las universidades del Consejo de Rectores presentan una madurez considerablemente mayor a las privadas en sus sitios Web, sin que la ubicación geográfica sea un factor relevante. 
Tabla 9. Cruce de tablas 5, 6, 7 y 8 .

\begin{tabular}{|c|c|c|c|c|c|c|c|c|c|}
\hline \multirow{3}{*}{ Universidades } & \multicolumn{6}{|c|}{ Provisión } & \multirow{2}{*}{\multicolumn{2}{|c|}{ Procesamiento }} & \multirow{3}{*}{ Total } \\
\hline & \multicolumn{2}{|c|}{ Nivel 1} & \multicolumn{2}{|c|}{ Nivel 2} & \multicolumn{2}{|c|}{ Nivel 3} & & & \\
\hline & Cantidad & $\%$ & Cantidad & $\%$ & Cantidad & $\%$ & Cantidad & $\%$ & \\
\hline CR en regiones & 0 & 0 & 0 & 0 & 1 & 5 & 19 & 95 & 20 \\
\hline CR en RM & 0 & 0 & 1 & 20 & 0 & 0 & 4 & 80 & 5 \\
\hline Priv. en regiones & 0 & 0 & 0 & 0 & 4 & 40 & 6 & 60 & 10 \\
\hline Priv. en RM & 0 & 0 & 0 & 0 & 9 & 36 & 16 & 64 & 25 \\
\hline
\end{tabular}

Para complementar esta conclusión se desarrolla a continuación el siguiente análisis.

\section{Análisis del nivel de adopción de las universidades clasificadas por tramos}

Hace algunos años el Gobierno presentó una clasificación de las universidades del Consejo de Rectores en cuatro tramos. Dichos tramos se elaboraron con el fin de determinar la casa de estudios líder de cada tramo como referente para fijar los precios o aranceles de las carreras del resto de las universidades pertenecientes al tramo. Con el fin de realizar esta clasificación el gobierno realizó un ranking de universidades, tomando en cuenta el valor de veinte carreras y analizando diversos factores como: retención de alumnos de primer año, tasa de alumnos titulados, cantidad de doctores y magíster con jornada completa, proyectos Fondecyt, entre otros.

En vista que dichos tramos pueden ser considerados como una herramienta objetiva de clasificación de las universidades pertenecientes al Consejo de Rectores y la importancia que adquirirán en el futuro, se realizó el siguiente análisis:

Tabla 10. Nivel de adopción de las universidades clasificadas por tramos.

\begin{tabular}{|c|c|c|c|c|c|c|c|c|c|}
\hline \multirow{3}{*}{ Tramos } & \multicolumn{6}{|c|}{ Provisión } & \multicolumn{2}{|c|}{ Proc. } & \multirow{3}{*}{ Total } \\
\hline & \multicolumn{2}{|c|}{ Nivel 1} & \multicolumn{2}{|c|}{ Nivel 2} & \multicolumn{2}{|c|}{ Nivel 3} & & & \\
\hline & ن & $b^{\circ}$ & نُ & $b^{\circ}$ & $\underset{ٍ}{\tilde{\Xi}}$ & 8 & ذٍ & $b^{\circ}$ & \\
\hline Tramo 1 & 0 & 0 & 0 & 0 & 0 & 0 & 3 & 100 & 3 \\
\hline Tramo 2 & 0 & 0 & 0 & 0 & 0 & 0 & 8 & 100 & 8 \\
\hline Tramo 3 & 0 & 0 & 0 & 0 & 2 & 25 & 6 & 75 & 8 \\
\hline Tramo 4 & 0 & 0 & 1 & 20 & 0 & 0 & 4 & 80 & 5 \\
\hline
\end{tabular}

Como se puede apreciar, las universidades clasificadas en los dos primeros tramos se encuentran en el nivel más alto de adopción de uso de Internet, ya que todas ellas alcanzan la etapa 3 de procesamiento, situación que se mantiene para el tramo 2 .
El nivel de adopción disminuye en los tramos posteriores, pero no en grandes porcentajes. En el tramo tres, el $75 \%$ de las universidades se encuentra en el nivel de procesamiento y el $25 \%$ en el nivel más alto de la etapa de provisión. A su vez, en el tramo 4 el $80 \%$ de las instituciones se encuentra en la etapa de procesamiento y el $20 \%$ en el segundo nivel de la etapa de provisión (ver tabla 10).

Los anteriores resultados podrían demostrar una congruencia del modelo eMICA al medir el nivel de desarrollo alcanzado por los sitios Web de dichas instituciones, ya que concuerdan los niveles más altos de adopción con las universidades clasificadas en los tramos más altos y viceversa.

Análisis del nivel de adopción de las universidades introduciendo nuevos atributos a la etapa 3 del modelo eMICA

Dado que en su mayoría las universidades chilenas han adoptado fuertemente el uso de Internet en sus funciones (el $75 \%$ se encuentran en la etapa 3), el modelo no permite determinar cuál es la mejor en este aspecto, ni realizar profundas comparaciones. Por lo anterior, en esta sección se analiza de manera más detallada la etapa 3 , para así poder reflejar los distintos tipos de transacciones desarrollados por las universidades.

Al realizar el estudio se determinó que además de las transacciones de consulta y financieras se dan otro tipo de transacciones propias de la naturaleza de las instituciones estudiadas, que se pueden clasificar como académicas $\mathrm{y}$ administrativas.

Por lo tanto, la propuesta apunta a que la etapa 3 (procesamiento) sea dividida en dos niveles. El primer nivel correspondería a transacciones de consulta y el segundo a transacciones de modificación.

\section{Nivel 1: Transacciones de consulta}

Interacción con el servidor: corresponden a transacciones de consulta, es decir, el usuario sólo puede acceder a la 
información, pero no puede realizar ninguna operación o cambio con ella. Dentro de estas transacciones se encuentra el acceso a la ficha personal, el historial de notas y las notas parciales, nómina de certificados solicitados, estado de deuda, entre otros.

\section{Nivel 2: Transacciones de modificación}

Este tipo de transacciones consisten en que el usuario no sólo puede acceder a la información, sino que también puede realizar operaciones dentro de la página Web.

- Transacciones académicas: inscripción de ramos, desarrollo de pruebas y controles en línea.

- Transacciones administrativas: solicitud de certificados, evaluación docente, modificación de la ficha personal.

- Transacciones financieras: pago online de matrículas, aranceles, cursos, seminarios, entre otros.

- Estado de orden: corresponde a cuando las transacciones anteriores cambian de estado, es decir, pedido, en trámite, entregado/evaluado.

Con esta segmentación en la etapa 3 se reflejan los diferentes niveles de procesamiento de los sitios Web de las universidades chilenas, evitando clasificarlas a todas dentro de la etapa 3, cuando en realidad sólo pueden realizar algunos tipos de transacciones.

Para explicar de forma más gráfica esta propuesta, a continuación se entregan los resultados obtenidos con la nueva clasificación.

Como se puede apreciar en tabla 11, el número de universidades que tienen disponible en su página transacciones de consulta son 38 , que corresponden a $63,3 \%$ del total de universidades; las que cuentan con pago online corresponden a 10 , es decir, un $16,7 \%$ del total; las que poseen transacciones académicas son 30 ,

Tabla 11. Resultados del modelo eMICA modificado.

\begin{tabular}{|c|c|c|c|c|c|}
\hline Etapa & Nivel & Atributos & $\begin{array}{l}\text { Nro } \\
\text { Univ. }\end{array}$ & $\begin{array}{c}\% \\
\text { Total }\end{array}$ & $\begin{array}{c}\% \\
\text { Etapa } 3\end{array}$ \\
\hline \multirow{5}{*}{ 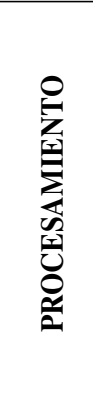 } & 1 & $\begin{array}{l}\text { Interacción con } \\
\text { el servidor }\end{array}$ & 38 & 63.3 & 84,4 \\
\hline & \multirow[t]{4}{*}{2} & \begin{tabular}{|l} 
Transacciones \\
académicas
\end{tabular} & 30 & 50,0 & 66,6 \\
\hline & & \begin{tabular}{|l|} 
Transacciones \\
administrativas
\end{tabular} & 22 & 36,7 & 48,8 \\
\hline & & $\begin{array}{l}\text { Transacciones } \\
\text { financieras }\end{array}$ & 10 & 16,7 & 22,2 \\
\hline & & $\begin{array}{l}\text { Estado de la } \\
\text { orden }\end{array}$ & 37 & 61,7 & 82,2 \\
\hline
\end{tabular}

que corresponde al 50\% del total, y las con transacciones administrativas son 22 , es decir, un $36,7 \%$ del total.

Por lo tanto, con esta clasificación se puede determinar qué tipo de transacciones son las que las universidades realizan a través de sus sitios Web, permitiendo un análisis más profundo, que revele la realidad de las universidades chilenas.

\section{OTROS RESULTADOS}

Las universidades chilenas ofrecen en sus sitios Web una gran variedad de servicios que van desde información estática que sirve principalmente para promover a la universidad con sus respectivas actividades, publicar noticias del acontecer nacional y de la institución en sí hasta procesos transaccionales de carácter administrativo, educativo y económico, como se detallan en la figura 3.

Dentro de esta gama de servicios se encuentran principalmente:

- Los formularios de consulta, que permiten a los usuarios comunicar sus dudas rápidamente.

- Catálogos de alto nivel, en el cual las universidades despliegan una página propia para cada carrera, lo que tiene como ventajas facilitar el acceso a la información, agrupando una mayor cantidad de ella en las páginas independientes, logrando entre otras cosas comunicar noticias que son del exclusivo interés de los estudiantes de la carrera, además de sus publicaciones e información sobre seminarios. Este atributo también considera a los catálogos propios para admisión que reúnen principalmente servicios orientados a postulantes como: links a contacto, pasos a seguir en las postulaciones, chat, entre otros.

- Ayuda al cliente en la forma de preguntas frecuentes y mapa del sitio. Las preguntas frecuentes, en su mayoría, están orientadas a postulantes y el mapa del sitio sirve para alumnos potenciales y actuales a encontrar lo que buscan dentro de la página de manera más fácil y rápida.

- Los buscadores son también un servicio de mucha utilidad para obtener de manera rápida lo que se busca. La diferencia entre éstos y el mapa del sitio es que en el mapa se encuentra el desglose de las secciones que conforman la página y los buscadores permiten determinar las secciones relacionadas con una palabra o frase clave, tanto dentro como fuera del sitio de la universidad.

- Valor agregado como bolsas de trabajo a las que los alumnos y ex alumnos pueden postular, ya sea para 
realizar la práctica profesional o buscar trabajo; un disco virtual, que consiste en un espacio en la red otorgado por la universidad a los alumnos para que ellos puedan almacenar cualquier tipo de archivo; cifras económicas, entre otras.

- Chat, que permite a los alumnos y funcionarios de la institución comunicarse en tiempo real.

- Foro, que permite a los usuarios expresar sus opiniones respondiendo a una pregunta o escribiendo sus comentarios.

- Noticias vía mail, que permiten mantener informadas a todas las personas que tienen una cuenta de correo de la universidad, respecto a noticias o actividades que se realizan, sin que tengan que visitar la página de la universidad.

- Opciones de lenguaje, cuya importancia radica en que permiten aprovechar las características globales de Internet.

- La posibilidad de realizar transacciones a través de la red es un servicio muy importante, ya que facilita y agiliza el pago de aranceles, cursos o seminarios y permite realizar de forma más rápida y menos burocrática actividades como: inscribir ramos, solicitar certificados, realizar la evaluación docente y pruebas online.

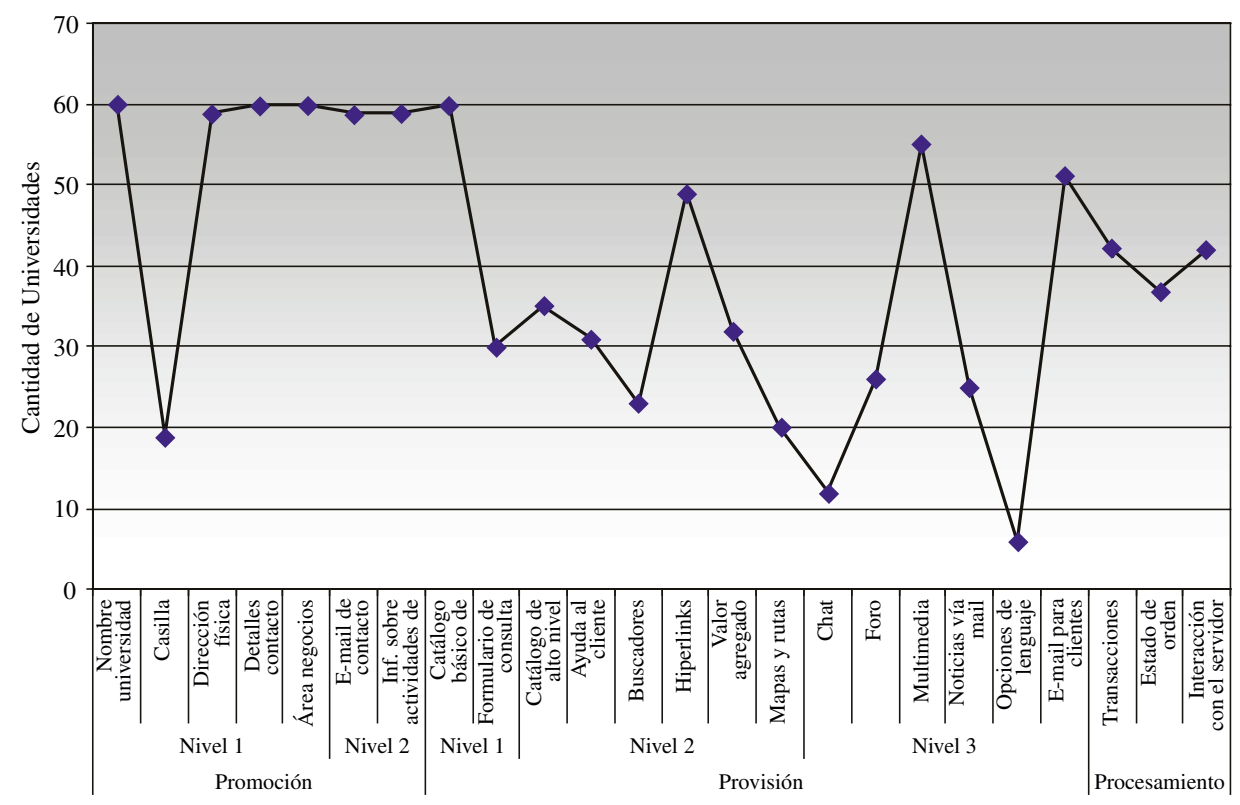

Figura 3. Gráfico de frecuencias.

\section{CONCLUSIONES}

Considerando la información analizada para el desarrollo de este estudio, es posible concluir que las universidades chilenas muestran un alto grado de adopción de Internet en sus sitios Web, ya que luego de la aplicación del modelo eMICA un $75 \%$ de ellas se encuentran en la etapa más alta (procesamiento), mientras que un $25 \%$ en la etapa 2 (provisión). Este análisis general permite determinar que las universidades chilenas han alcanzado un alto nivel de madurez en sus sitios Web, lo cual se puede relacionar con la alta competitividad a la que dichas instituciones se han visto expuestas los últimos 20 años.
A raíz de lo antes señalado, en la actualidad los servicios prestados por las universidades a través de sus sitios ya no hacen necesario que los alumnos actuales y potenciales tengan que acercarse a una oficina para obtener información.

Luego de la utilización del modelo eMICA en el ámbito universitario es posible señalar su alto grado de aplicabilidad a esta actividad, sumándose así un sector adicional sobre los cuales el modelo ya ha demostrado ser eficaz.

Considerando un análisis más detallado de los resultados se desprende que: 
- 35 universidades son de carácter privado, de las cuales $13(37,1 \%)$ se encuentran en la etapa de provisión y $22(62,9 \%)$ en la etapa de procesamiento.

- Las 25 restantes pertenecen al Consejo de Rectores, alcanzando sólo 2 (8\%) de ellas la etapa 2 y 23 (92\%) la etapa 3.

- 30 universidades se encuentran en la Región Metropolitana de Santiago, de ellas 10 (33,3\%) alcanzan la etapa 2 y $20(66,7 \%)$ alcanzan la etapa 3.

- 30 universidades se encuentran en regiones, de las cuales $5(16,7 \%)$ se encuentran en el nivel 3 de la etapa 2 y $25(83,3 \%)$ en la etapa 3 .

Del cruce de los resultados anteriores se obtiene que:

- De las universidades del Consejo de Rectores, 20 se encuentran en regiones, de las cuales 19 (95\%) alcanzan la etapa 3 y sólo 1 (5\%) la etapa 2 nivel 3. Las 5 restantes se encuentran en la Región Metropolitana, de las cuales $4(80 \%)$ se encuentran en la etapa 3 y sólo 1 (20\%) en la etapa 2 nivel 2.

- Del total de universidades privadas, 10 se encuentran en regiones, de ellas $6(60 \%)$ alcanzan la etapa 3 y $4(40 \%)$ la etapa 2 nivel 3 . Las 25 restantes se encuentran en la Región Metropolitana, de las que $16(64 \%)$ se encuentran en la etapa 3 y $9(36 \%)$ en la etapa 2 nivel 3 .

Por lo tanto, las universidades pertenecientes al Consejo de Rectores alcanzan un nivel mayor de desarrollo en sus sitios Web que las universidades privadas, no siendo una variable relevante para este punto la ubicación geográfica.

\section{RECOMENDACIONES Y LIMITACIONES}

Para un estudio posterior sería muy interesante considerar en la metodología las siguientes recomendaciones y limitaciones:

- El modelo eMICA tiene como principal desventaja que sólo mide la presencia o ausencia de un servicio, pero no si es fácil de encontrar o el tiempo para acceder a él, es decir, no evalúa la usabilidad de la página.

- De acuerdo a la estructura del modelo es difícil establecer qué universidad tiene el mejor desempeño. Para solucionar esto sería necesario ampliar la etapa 3 de procesamiento a dos o más niveles que permitan determinar con mayor certeza el nivel de adopción exacto de cada institución.
- Los servicios de los sitios Web universitarios están en constante cambio, lo cual implica que los resultados obtenidos pueden ir variando según el momento en que el estudio se lleve a cabo.

\section{REFERENCIAS}

[1] M. Beller and E. Or. "The crossroads between lifelong learning and information technology: A challenge facing leading universities". Journal of Computer Mediated Communication. Vol. 4 $\mathrm{N}^{\circ} 2.1998$.

[2] L. Burgess and J. Cooper. "MICA: Model of Internet Commerce Adoption”. S. Rahman and M. Raisinghani. Electronic Commerce: Opportunities and Challenges. Idea Group Publising. USA. 2000.

[3] L. Burgess and J. Cooper. "Extending the Viability of MICA (Model of Internet Commerce Adoption) as a Metric for Explaining the Process of Business Adoption of Internet Commerce". International Conference on Telecommunications and Electronic Commerce. Dallas. November. 2000.

[4] L. Burgess, N. Cerpa, J. Cooper and Jason Sargent. "To what extent are Chilean Regional Tourism Organizations (RTOs) leveraging web technologies to promote regional tourism". Proceedings of Collaborative Electronic Commerce Technology and Research (CollECTeR LatAm 2005). Talca, Chile. October 3-5, 2005.

[5] L. Burgess, J. Cooper, N. Cerpa and J. Sargent. "A comparative analysis of the use of the web for destination marketing by regional tourism organizations in Chile and the Asia Pacific". CollECTeR Conference. Sydney, Australia. 16 June 2005.

[6] Consejo de Rectores. "Información de Universidades pertenecientes al Consejo de Rectores". Santiago, Chile. 2005. Fecha de consulta: 10 noviembre de 2005. Disponible en: http://www.cruch.cl/

[7] L. DeConti. "Planning and Creating a Government Web Site: Learning from the Experience of US States". Information Systems for Public Sector Management. Working Paper Series $\mathrm{N}^{\circ} 2$. Institute for Development Policy and Management. University of Manchester, 1998. Fecha de consulta: 11 de 
octubre de 2005. Disponible en: http://www.sed. manchester.ac.uk/idpm/research/publications/wp/ igovernment/igov_wp02.htm

[8] B. Doolin, L. Burgess, J. Cooper and C. Alcock. "Internet Commerce Adoption by New Zealand and Australian Regional Tourism Organisations: A Comparative Study Using eMICA". Proceedings ECECR-4. Dallas, Texas. November 8-11, 2001.

[9] P. Evans and T. Wurster. "Strategy and the New Economics of Information". Harvard Business Review. Issue September-October, pp. 71-82. 1997.

[10] S. Godoy and S. Herrera. "Internet usage in Chile and the world: First results of the World Internet ProjectChile". 2004. Fecha de Consulta: 1 de septiembre de 2005. Disponible en: http://www.wipchile.cl

[11] S. Godoy and S. Herrera. "Pilot study WIP Chile 2003". Media Research Institute - Institute of Sociology in cooperation with Centro de Estudio de la Economía Digital, Cámara de Comercio de Santiago. Fecha de consulta: 1 de Septiembre de 2005. Disponible en: http://www.wipchile.cl

[12] A. Hartman and J. Sifonis. "Net Ready. Estrategias para el éxito en la Economía". Primera edición. Editorial McGraw-Hill. 2000.

[13] M. Hitt and R. Ireland. "Administración estratégica. Competitividad y Conceptos de Globalización”. Tercera Edición. Thompson editors. 1998.

[14] B. Ives and S. Jarvenpaa. "Will Internet Revolutionize Business Education and Research?". Sloan Management Review. Spring. 1996.
[15] S.L. Jarvenpaa and B. Ives. "Introducing Transformational Information Technologies: The Case of the World Wide Web Technology". International Journal of Electronic Commerce. Vol. $1 \mathrm{~N}^{\mathrm{o}}$ 1, pp. 95-126. 1996.

[16] K. Kiser. "10 Things We Know So Far About Online Training”. Training. Vol. 36 N$^{\mathrm{o}}$ 11, pp. 66-74. 1999.

[17] R. Lenzner and S.S. Johnson. "Seeing things as they really are". Forbes. Fecha de Consulta: 20 de junio de 2005. Disponible en: http://www. forbes. com/forbes/97/0310/5905122a.htm

[18] G. Piccoli, R. Ahmad and B. Ives. "Web-based virtual learning environment". MIS Quarterly, Vol. $25 \mathrm{~N}^{\circ}$ 4, pp. 401-426. December 2001.

[19] C. Plana, N. Cerpa and P. B. Bro. "Bases para la Creación de una Metodología de Adopción de Comercio Electrónico para las PYMES Chilenas". Revista de la Facultad de Ingeniería - Universidad de Tarapacá. Vol. 14 Nº 1, pp. 49-63. 2006.

[20] J.A. Quelch and L.R. Klein. "The Internet and International Marketing”. Sloan Management Review, Spring, pp. 60-75. 1996.

[21] J. Rayport and B. Jaworsky. "E-Commerce". Primera edición. McGraw-Hill. 2003.

[22] C.T. Romm and J. Wong. "The Dynamics of Establishing Organizational Web Sites: Some Puzzling Findings". Australian Journal of Information Systems. Vol. $5 \mathrm{~N}^{\circ}$ 2, pp. 60-68. 1998. 